\title{
Generation of half-space sound fields with application to personal sound systems.
}

\author{
M. A. Poletti \\ Callaghan Innovation, P.O. Box 31-310, Lower Hutt, New Zealand \\ F. M. Fazi \\ Institute of Sound and Vibration Research, University of Southampton, SO17 1BJ, \\ United Kingdom
}

Running title: Half-space sound fields

\begin{abstract}
A method is presented for generating a sound field that is significantly attenuated over half of the reproduction region, which has application to the generation of two independent sound fields for two listeners. The half-space sound field is produced by attenuating the negative or positive modes in the cylindrical or spherical expansion of a plane wave or point source sound field. It is shown that this is equivalent to adding to the original sound field, in quadrature, a second field which is the Hilbert transform of the original field. The resulting analytic field has a small magnitude in one half of the plane. Methods are presented for controlling the attenuation in the unwanted halfspace. Finally, a simulation is presented showing the generation of a wideband pulse that propagates across half of the area within a circular array of sources.
\end{abstract}

PACS numbers 43.60.Fg, 43.38.Md, 43.60.Tj 


\section{INTRODUCTION}

A recent topic of interest in sound reproduction systems is personal sound systems, which aim to provide independent sound fields for separate listeners ${ }^{1,2}$. A number of methods have been applied to producing multiple zones with independent sound fields, using a single set of loudspeakers, such as beamforming ${ }^{1,3}$, pressure matching ${ }^{4,5}$, mode matching ${ }^{6}$ and optimal contrast ${ }^{7-10}$. The optimal contrast methods maximize the ratio of potential energy in a desired zone to that in an unwanted zone and as a consequence do not specify a direction of propagation. This allows the solution to choose a direction of propagation that allows the greatest contrast. A combination of optimal contrast with the requirement of a planar sound field was used in ${ }^{11}$ to produce a trade-off between the maximum contrast and a desired sound direction.

Methods have also been investigated that control the radiation pattern of an array of sources, subject to controlling sound levels in a target zone, to minimize the radiated sound power ${ }^{12,13}$. A recent approach to producing personal sound for two listeners is to create a sound field with a null (i.e. a region with low sound pressure level) centered at one listener ${ }^{14}$. This produces a desired sound field for the other listener and ensures there is very low interference at the position of the first listener. By linearity a second field can then be produced for the first listener with a null at the second listener position. The nulls are produced by suppressing the low-order modes in the cylindrical or spherical mode expansions of the sound field at each listener position.

Many of these approaches provide a set of relatively small zones of sound reproduction each of which is sufficient for a single listener. It would be an advantage to provide a sound zone that extends over a wider area that could be sufficiently large to enclose multiple listeners. This paper presents a method of achieving this. The method generates a sound field that exists over half of the area within a multi-channel sound reproduction system. This is possible if the direction of propagation of the sound is parallel to the boundary between the two half-spaces. It will be shown that the half space field is produced by suppressing the positive or negative index modes in the cylindrical or spherical harmonic expansions of the sound field ${ }^{14}$. Simulations will be presented that show that wideband sound fields can be created within a half- 
space. If the half-space requirement is relaxed, limited directionality can be produced with silence in a more restricted region.

The structure of this paper is as follows. In section 2 the theory describing the Hilbert transform of a 2D sound field is presented. Section 3 presents methods for controlling the characteristics of the field and considers half-space fields for the 3D case. Finally, section 4 presents simulations showing the generation of two independent, wideband, half-space fields.

\section{THEORY}

\section{A. Hilbert transform of a 2D sound field}

Consider the general solution to the 2D homogeneous Helmholtz equation in polar coordinates $\mathbf{r}=(R, \phi)$ represented by the Herglotz wave function ${ }^{15}$

$$
p(R, \phi, k)=\frac{1}{2 \pi} \int_{0}^{2 \pi} A\left(\phi^{\prime}\right) e^{i k R \cos \left(\phi-\phi^{\prime}\right)} d \phi^{\prime} .
$$

where $k=\omega / c$ is the wavenumber at radian frequency $\omega, c$ is the speed of sound and $A\left(\phi^{\prime}\right)$ is the plane wave angular amplitude distribution at wavenumber $k . \phi^{\prime}$ indicates the direction of arrival of the plane waves and the positive time convention $e^{i \omega t}$ is used throughout the paper. Since the angular distribution is periodic it can be written

$$
A\left(\phi^{\prime}\right)=\sum_{m=-\infty}^{\infty} \alpha_{m} e^{i m \phi^{\prime}}
$$

The Jacobi Anger expansion of the plane wave term is ${ }^{16}$

$$
e^{i k R \cos \left(\phi-\phi^{\prime}\right)}=\sum_{m=-\infty}^{\infty} i^{m} J_{m}(k R) e^{i m\left(\phi-\phi^{\prime}\right)},
$$

where $J_{m}($.$) is the m$ th Bessel function of the first kind ${ }^{16}$. Substituting this expansion and (2) into (1), rearranging the order of sum and integration and applying the orthogonality relation of complex exponential functions yields

$$
p(R, \phi, k)=\sum_{m=-\infty}^{\infty} \alpha_{m} i^{m} J_{m}(k R) e^{i m \phi},
$$

which has the standard form of the interior 2D solution to the wave equation with coefficients $a_{m}=\alpha_{m} i^{m}$. For a finite radius $R$ and wave number $k$, the expansion order 
can be limited to $m \in[-M, M]$ where $M=\lceil k R\rceil$ and where $\lceil$.$\rceil denotes rounding to$ the next highest integer ${ }^{17}$.

Consider multiplying the coefficients $\alpha_{m} i^{m}$ by the step function sequence

$$
\mu_{m}=\left\{\begin{array}{cc}
0, & m<0 \\
1, & m=0 . \\
2, & m>0
\end{array}\right.
$$

Note that this sequence can be written as

$$
\mu_{m}=1+\operatorname{sgn}(m),
$$

where sgn is the signum function

$$
\operatorname{sgn}(m)=\left\{\begin{array}{c}
-1, m<0 \\
0, m=0 . \\
1, m>0
\end{array}\right.
$$

The angular distribution produced by eliminating the negative mode amplitudes is, from (2), the convolution of $A\left(\phi^{\prime}\right)$ with the function

$$
U\left(\phi^{\prime}\right)=\sum_{m=-\infty}^{\infty} \mu_{m} e^{i m \phi^{\prime}} .
$$

It can be shown by taking limits (Appendix A) that

$$
U\left(\phi^{\prime}\right)=\delta\left(\phi^{\prime}\right)+\frac{i}{\tan \left(\phi^{\prime} / 2\right)} .
$$

The angular distribution therefore becomes

$$
\begin{aligned}
\hat{A}\left(\phi^{\prime}\right) & =A\left(\phi^{\prime}\right) *\left[\delta\left(\phi^{\prime}\right)+\frac{i}{\tan \left(\phi^{\prime} / 2\right)}\right] \\
& =A\left(\phi^{\prime}\right)+i\left[A\left(\phi^{\prime}\right) * \frac{1}{\tan \left(\phi^{\prime} / 2\right)}\right],
\end{aligned}
$$

which is the original distribution plus the quadrature addition of the function

$$
\tilde{A}\left(\phi^{\prime}\right)=\int_{0}^{2 \pi} \frac{A\left(\phi^{\prime}-\theta\right)}{\tan (\theta / 2)} d \theta,
$$

which is the Hilbert transform of the plane wave distribution ${ }^{18}$ with associated sound field expansion

$$
\tilde{p}(R, \phi, k)=\sum_{m=-\infty}^{\infty} \operatorname{sgn}(m) \alpha_{m} i^{m} J_{m}(k R) e^{i m \phi} .
$$

The total sound field is 


$$
\hat{p}(R, \phi, k)=p(R, \phi, k)+i \int_{0}^{2 \pi} \tilde{A}\left(\phi^{\prime}\right) e^{i k R \cos \left(\phi^{\prime}-\phi\right)} d \phi^{\prime},
$$

which, in analogy with the analytic signal ${ }^{19}$, may be termed the analytic sound field, with expansion

$$
\hat{p}(R, \phi, k)=\alpha_{0} J_{0}(k R)+2 \sum_{m=1}^{\infty} \alpha_{m} i^{m} J_{m}(k R) e^{i m \phi} .
$$

\section{B. Half-space fields for a plane wave}

For the simple case of a single plane wave propagating along the negative $x$-axis, $A\left(\phi^{\prime}\right)=\delta\left(\phi^{\prime}\right), \alpha_{m}=1$, the Hilbert transform is $\tilde{A}\left(\phi^{\prime}\right)=1 / \tan \left(\phi^{\prime} / 2\right)$ and the resulting analytic field is

$$
\hat{p}(R, \phi, k)=p(R, \phi, k)+\tilde{p}(R, \phi, k),
$$

where $\tilde{p}(R, \phi, k)$ is the sound field associated with the Hilbert transform

$$
\tilde{p}(R, \phi, k)=i \int_{0}^{2 \pi} \frac{e^{i k R \cos \left(\phi^{\prime}-\phi\right)}}{\tan \left(\phi^{\prime} / 2\right)} d \phi^{\prime}=\sum_{m=-\infty}^{\infty} \operatorname{sgn}(m) i^{m} J_{m}(k R) e^{i m \phi} .
$$

By separating the positive and negative $m$ terms this can be put in the form

$$
\tilde{p}(R, \phi, k)=2 i \sum_{m=1}^{\infty} i^{m} J_{m}(k R) \sin (m \phi) .
$$

It is interesting to compare this sound field with that of the original plane wave, which has the well-known expansion ${ }^{20}$

$$
p(R, \phi, k)=J_{0}(k R)+2 \sum_{m=1}^{\infty} i^{m} J_{m}(k R) \cos (m \phi) .
$$

The Hilbert transform field thus has an expansion which is in phase and angular quadrature to the original sound field expansion. The analytic sound field is the sum of (17) and (18),

$$
\hat{p}(R, \phi, k)=J_{0}(k R)+2 \sum_{m=1}^{\infty} i^{m} J_{m}(k R) e^{i m \phi},
$$

which is the original field with negative modes suppressed, as expected.

The Hilbert transform field tends to produce a plane wave field similar to the original, but with a phase shift of 180 degrees in the upper half plane. Defining

$$
\tilde{p}(R, \phi, k)=p(R, \phi, k) \psi(R, \phi, k),
$$

the relative complex amplitude function $\psi(R, \phi, k)$, calculated using a finite 
expansion order of 40 in (16) and (17), is shown in Figure 1 for $k R=10$ and $k R=30$. The phase of the field is approximately offset by $\pi$ in the upper half plane $\phi \in[0, \pi]$ and the magnitude has additional ripple and is zero at 180 degrees, at the boundary between the two regions. A similar phase shift occurs at all frequencies but the transition is more rapid at higher $k R$ values and displays more rapid oscillations with angle.

The complex sum of the two fields (15) thus tends to produce a plane wave of twice the magnitude in the lower half space and an attenuated field in the lower half space. In the same manner, subtracting the Hilbert transform (suppressing the positive mode amplitudes),

$$
\hat{p}_{U}(R, \phi, k)=p(R, \phi, k)-\tilde{p}(R, \phi, k),
$$

tends to produce an upper half space field.
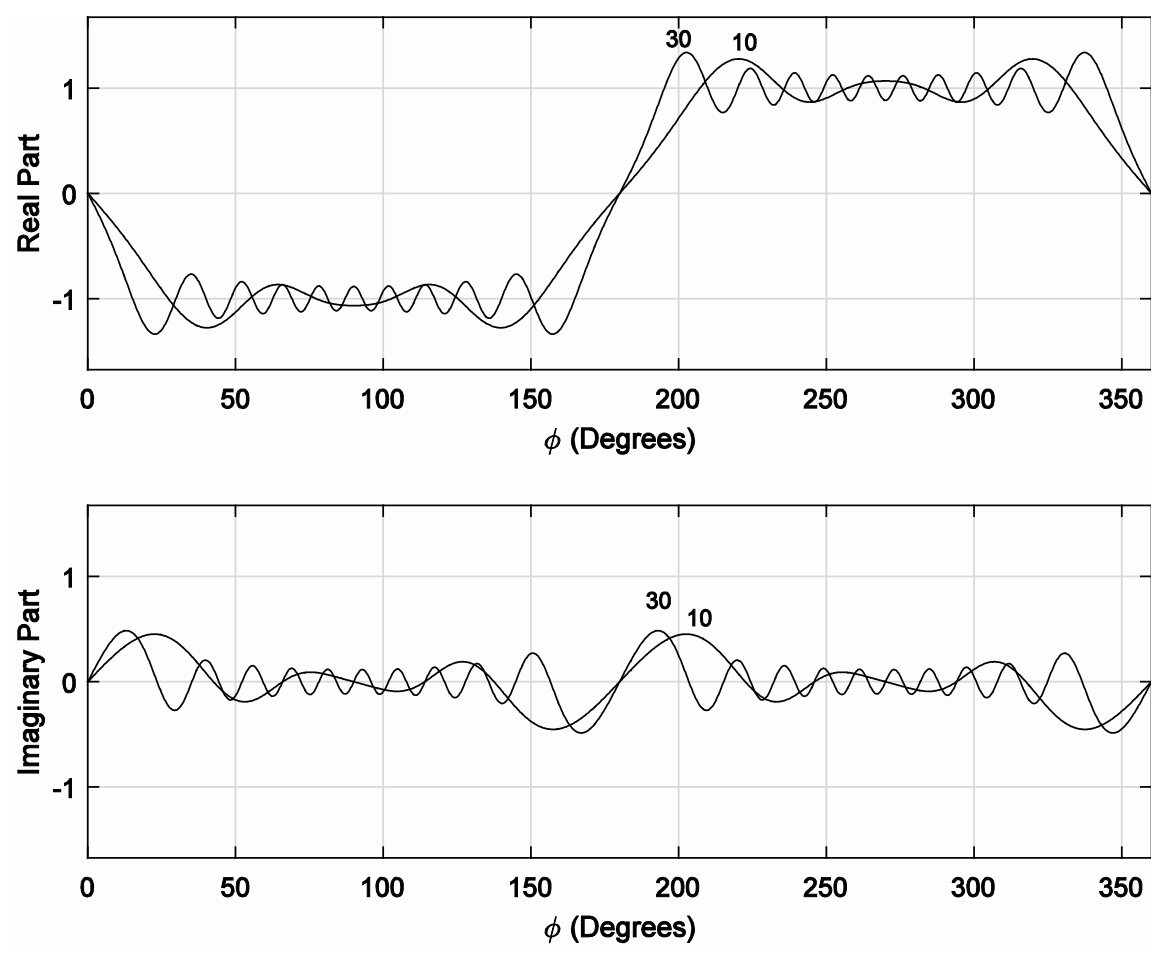

Figure 1: $\psi(R, \phi, k)$ for $k R=10$ and $k R=30$ 


\section{METHODS FOR CONTROLLING THE PROPERTIES OF HALF SPACE FIELDS}

\section{A. Reducing artefacts}

The sound field with the weighting given by (5) is shown in Fig. 2, for a frequency of $1 \mathrm{kHz}$, using a finite expansion order $M=\lceil k \sqrt{2} D\rceil=53$ where $D=2$. The field magnitude is relatively small in the upper half plane, but is not zero. This corresponds to the fact that the relative complex amplitude oscillates around the required value of -1 in Fig. 1. There is also ripple in the wave fronts in the lower half plane, also corresponding to oscillations in $\psi(R, \phi, k)$. These artefacts are caused by the sharp transition of the signum function through $m=0$. Similar artefacts occur when generating analytic signals where the corresponding analytic filter is required to have a step transition through zero $\mathrm{Hz}^{21}$.

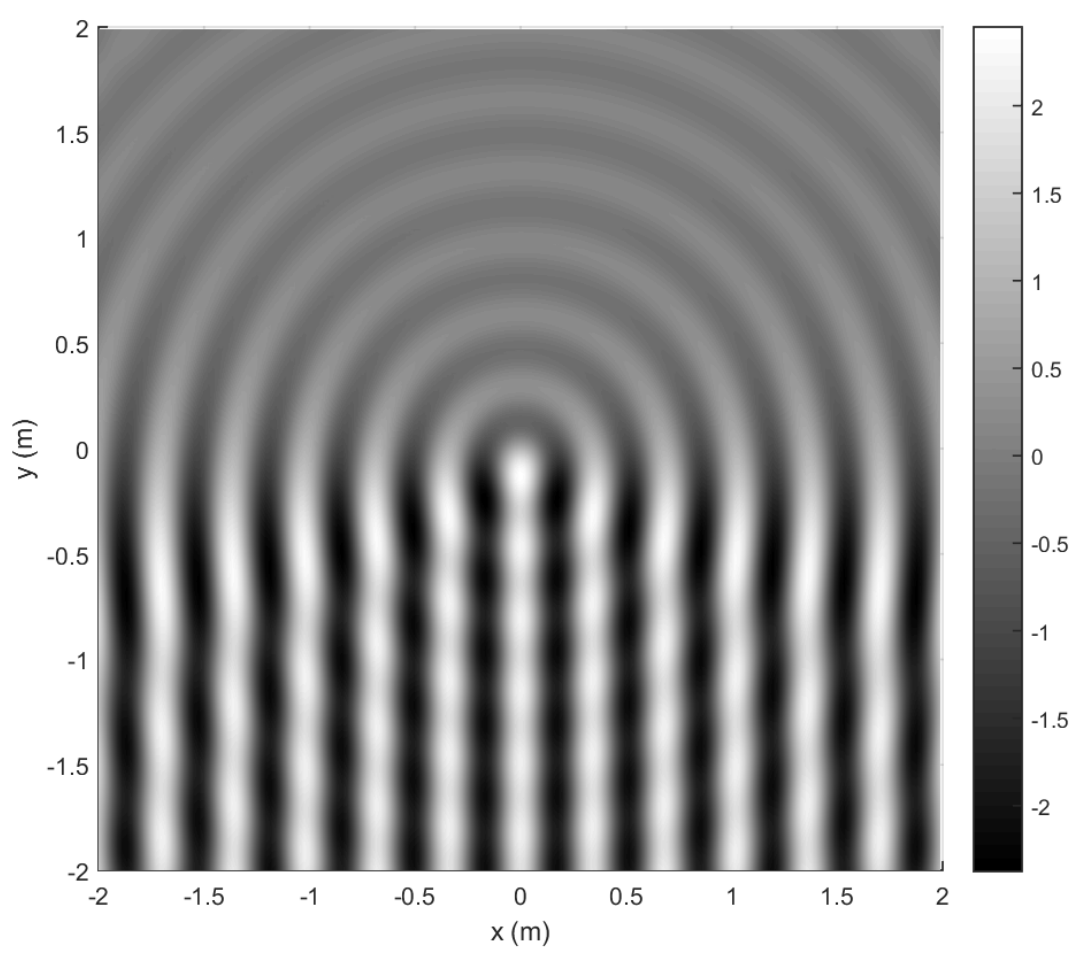

Figure 2: Lower half-space field produced at $1 \mathrm{kHz}$ for a step function weighting

The results can be improved by providing a smooth transition through $m=0$. This can be achieved by convolving the sequence $\mu_{m}$ with a smoothing filter $g_{m}$ which multiplies the spectrum $U\left(\phi^{\prime}\right)$ by the low-pass response $G\left(\phi^{\prime}\right)$, producing the modified spectrum (from (8) and (9)) 


$$
U_{g}\left(\phi^{\prime}\right)=\delta\left(\phi^{\prime}\right)+\frac{i G\left(\phi^{\prime}\right)}{\tan \left(\phi^{\prime} / 2\right)},
$$

where it is assumed that $G(0)=1$. The convolution has no effect on the real part but reduces the amplitudes of the imaginary part for $\left|\phi^{\prime}\right|>0$.

In practice it is simpler to directly generate a smoothed step sequence. For example, defining a transition length $N_{T}$, the function

$$
\mu_{m, N_{T}}=\left\{\begin{array}{c}
0, m<-\left(N_{T}-1\right) / 2 \\
1+\sin \left(m \pi / N_{T}\right), m \in\left[-\left(N_{T}-1\right) / 2,\left(N_{T}-1\right) / 2\right] \\
2, m>\left(N_{T}-1\right) / 2
\end{array}\right.
$$

produces a sinusoidal transition through $m=0$ with transition width $N_{T}$. The resulting plane wave relative amplitude $\psi(k, R, \phi)$ is shown in Fig. 3 for $N_{T}=15$, and for $k R=10$ and $k R=30$. The ripple is reduced compared to Fig. (1). The corresponding lower half space field at $1 \mathrm{kHz}$ is shown in Fig. 4. The amplitude of the sound field in the upper half space has a lower amplitude compared to Fig. (2), but is still not zero at all angles between 0 and 180 degrees.
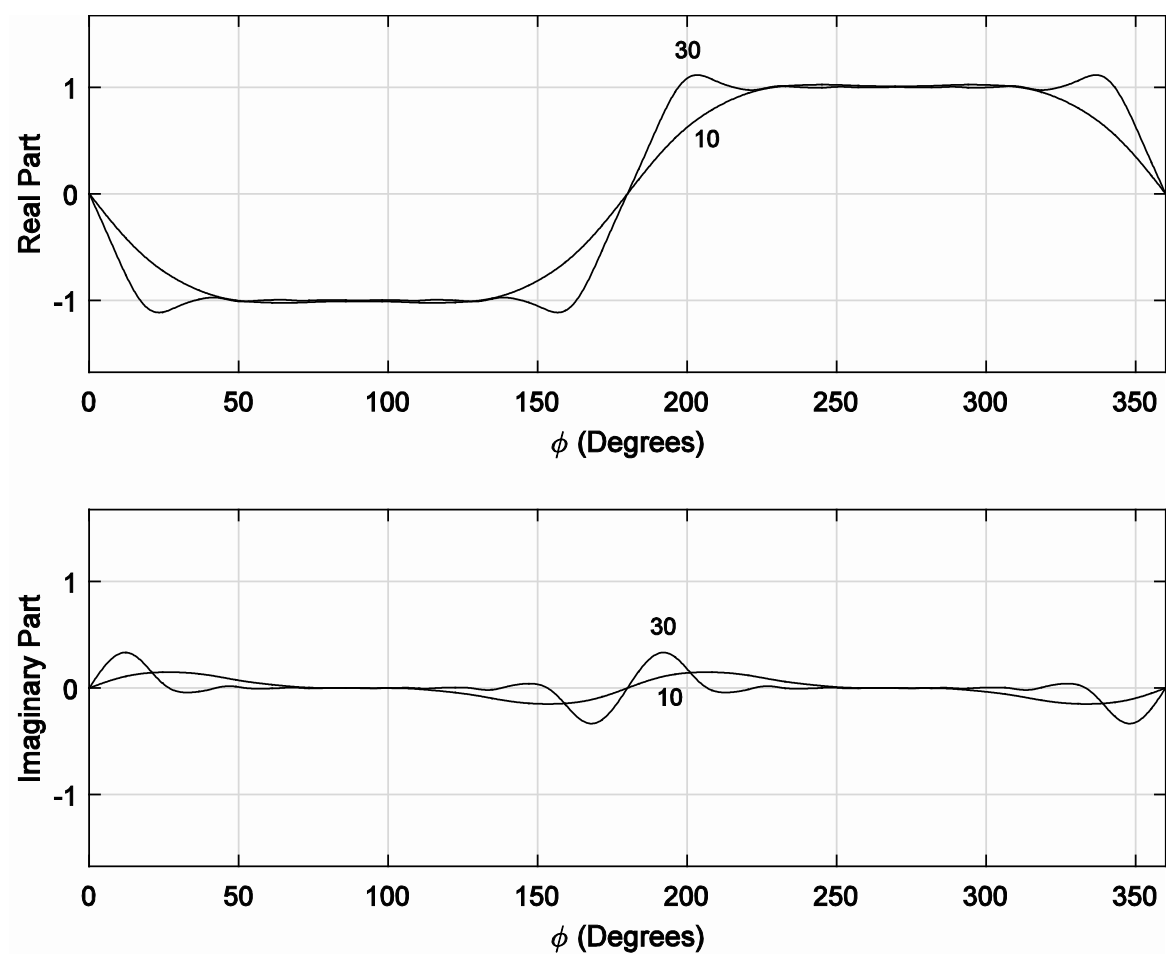

Figure 3: $\psi(R, \phi, k)$ for $k R=30, N_{T}=15$ transition width 


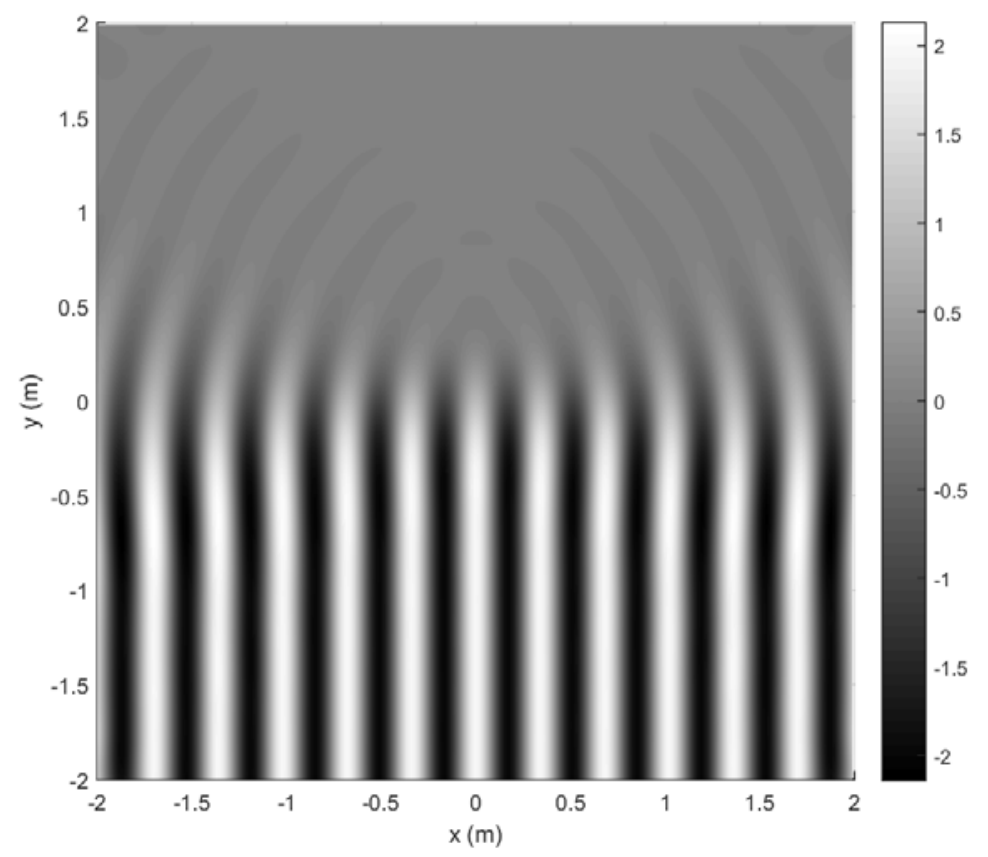

Figure 4: Lower half-space field produced at $1 \mathrm{kHz}$ for $N_{T}=15$ transition width

\section{B. Half space fields for more complex fields}

The examples above are for a single plane wave. The Hilbert transform of an arbitrary field as in (12) does not produce a half-space field. A plane wave with arbitrary angle of incidence $\phi_{i}$ will produce a half space with transition boundary given by the line $y=\tan \left(\phi_{i}\right) x$. Therefore a sum of plane waves will produce a sum of half spaces with different transition boundaries. However, fields can be generated with a limited range of angles of incidence and still produce a significant zone of silence. For example, Fig. 5 shows the magnitude in $\mathrm{dB}$ for a field consisting of a sum of three plane waves with equal amplitudes and incidence angles $-30,0$ and 30 degrees. The zone of silence is reduced approximately to $y<-\tan (\pi / 6)|x|$ compared to Fig. 4, but a useful area of silence is still produced. Hence, fields with a limited range of directionality, such as produced by stereo reproduction systems, can be produced over a region less than a half-space. 


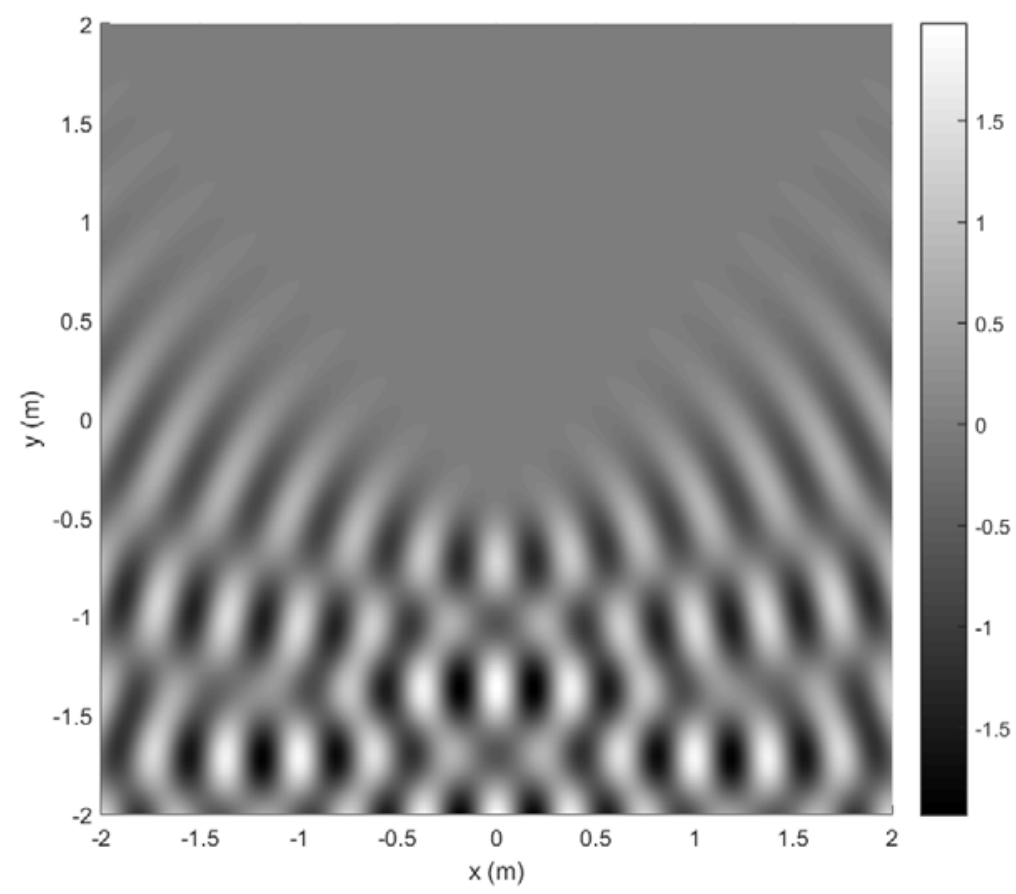

Figure 5: Half-space field at $1 \mathrm{kHz}$ for three plane waves of equal amplitude with incidence angles $-30,0$ and 30 degrees, with $\mathrm{N}_{\mathrm{T}}=15$ and offset 10

A half space can also be generated for a 2D point source. For example the 2D Green's function with source position $\mathbf{r}_{S}=\left(R_{S}, \phi_{S}\right)$ has the interior expansion ${ }^{22}$

$$
p_{S}(R, \phi, k)=H_{0}\left(k\left|\mathbf{r}-\mathbf{r}_{S}\right|\right)=\sum_{m=-\infty}^{\infty} J_{m}(k R) H_{m}\left(k R_{S}\right) e^{i m\left(\phi-\phi_{S}\right)}, R<R_{S},
$$

where $H_{m}(\bullet)$ is the $m$-th order Hankel function of the second kind ${ }^{16}$. (We ignore the additional $-i / 4$ factor in the Greens function which is not required.) The analytic field (14) with $\alpha_{m}=i^{-m} H_{m}\left(k R_{S}\right) \exp \left(-i m \phi_{S}\right)$ is a half space point source field with half space boundary $y=\tan \left(\phi_{S}\right) x$. An example is given in Fig. 6 for a line source at an angle of $\phi_{S}=45$ degrees and at radius $R_{S}=4 \mathrm{~m}$. 


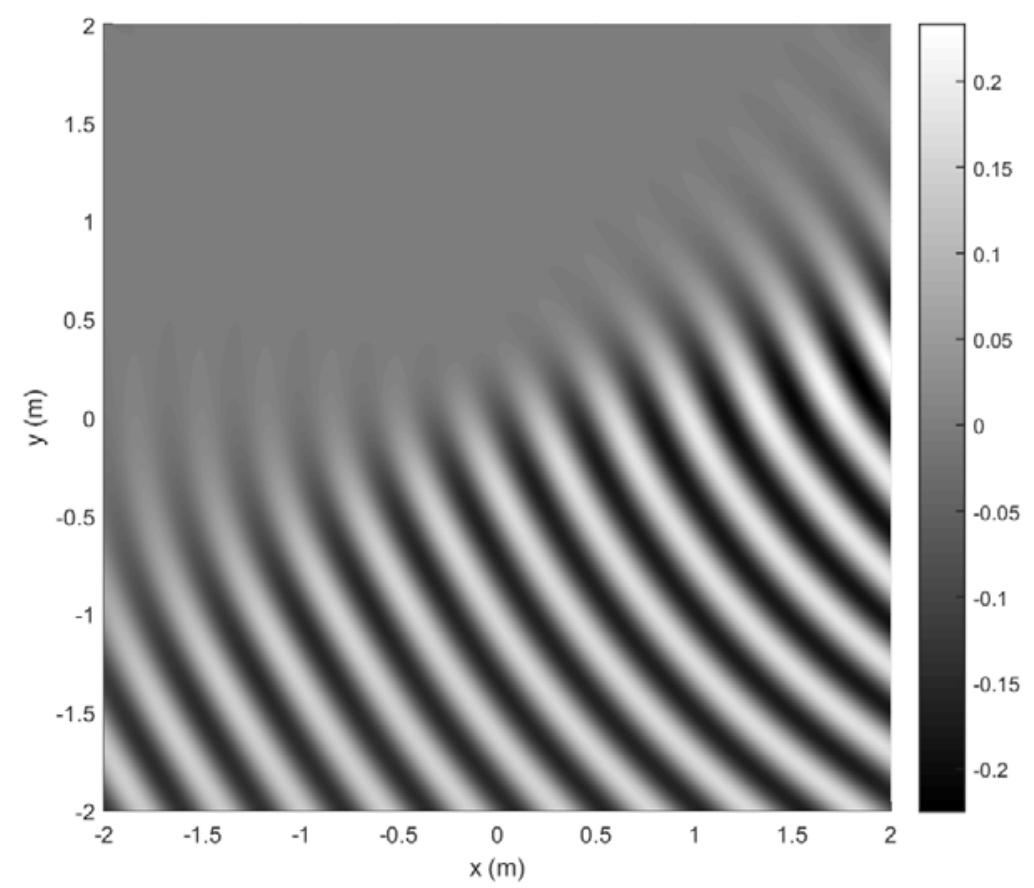

Figure 6: Half-space field at $1 \mathrm{kHz}$ for a line source at $\left(R_{s}, \phi_{s}\right)=(4, \pi / 4), N_{T}=15$

\section{Shifting the half-space boundary using offset mode weighting}

The half space fields in Figures 4 and 6 do not reduce to zero at the half-space boundary $(y=0)$ and intrude slightly into the zero-field half space. This intrusion may be reduced by shifting the sound field by an offset $y_{0}$. The sound field expansion coefficients for a translation of the sound field by an arbitrary offset $\left(R_{0}, \phi_{0}\right)$ are those obtained for a shift of the origin by $\left(R_{0}, \phi_{0}+\pi\right)$. Using the addition theorem, it can be shown that the coefficients of the translated field, $\vec{\alpha}_{m}$, are given by ${ }^{6}$

$$
i^{m} \vec{\alpha}_{m}=\sum_{n=-\infty}^{\infty} i^{n} \alpha_{n} J_{n-m}\left(k R_{0}\right) e^{i(n-m)\left(\phi_{0}+\pi\right)},
$$

which is the convolution of the original coefficients with the sequence

$$
J_{m}\left(k R_{0}\right) \exp \left(i m\left(\phi_{0}+\pi\right)\right) \text {, }
$$

$$
i^{m} \vec{\alpha}_{m}=i^{m} \alpha_{m} *\left[J_{m}\left(k R_{0}\right) e^{i m\left(\phi_{0}+\pi\right)}\right] .
$$

The resulting coefficients are shown in Figure 7(c) for $R_{0}=0.5$ and $\phi_{0}=-\pi / 2$ (a shift of $y_{0}=-0.5 \mathrm{~m}$ ). The effect of the convolution is to shift the mode weighting by an offset of approximately $m_{0}=k R_{0}$. The sound field translation may be more efficiently implemented by simply offsetting the mode weighting function. 

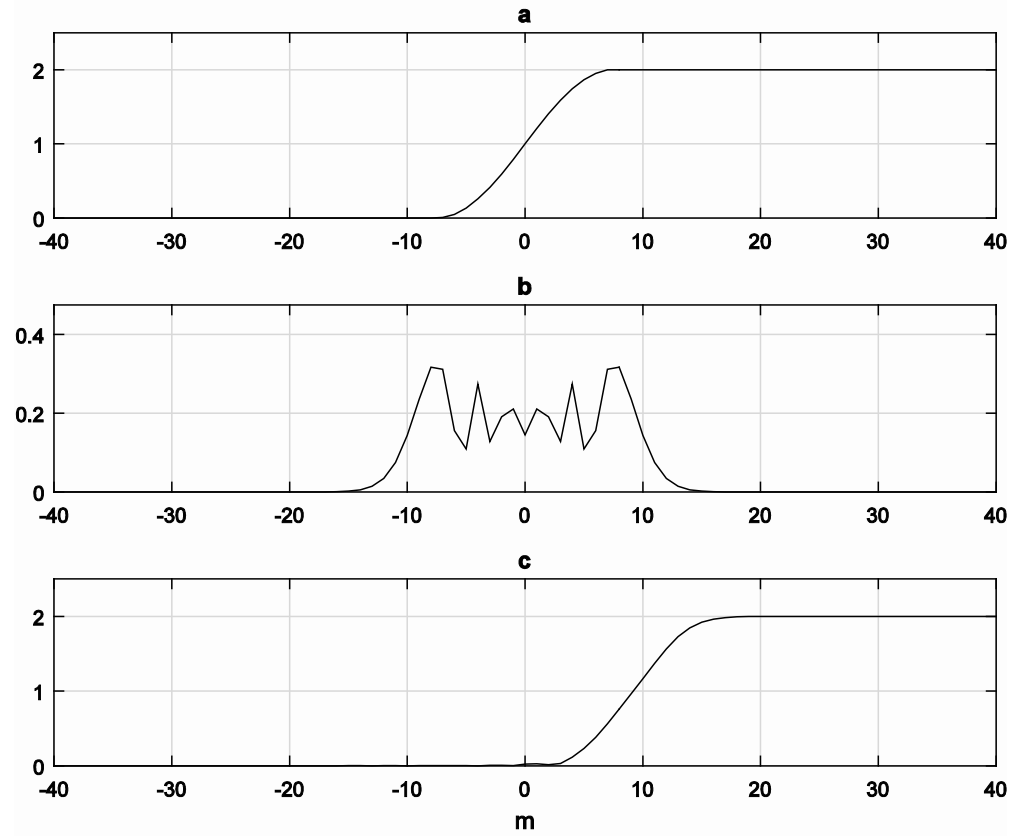

Figure 7: Coefficients for a $1 \mathrm{kHz}$ half space field (a), Bessel coefficients (b) and the coefficients obtained by the convolution of the two for a translation of $-0.5 \mathrm{~m}$ in y, (c)

For example, the magnitude of the half-space sound field in $\mathrm{dB}$ produced for the same mode weighting as in Fig. 4 with the transition centred at $m_{o}=0$ and $m_{o}=10$ are shown in Figures 8 and 9. The transition region is shifted in the negative $y$ direction by $R_{o}=0.5 \mathrm{~m}$ by the offset. Hence, the overlap between two half-spaces can be reduced.

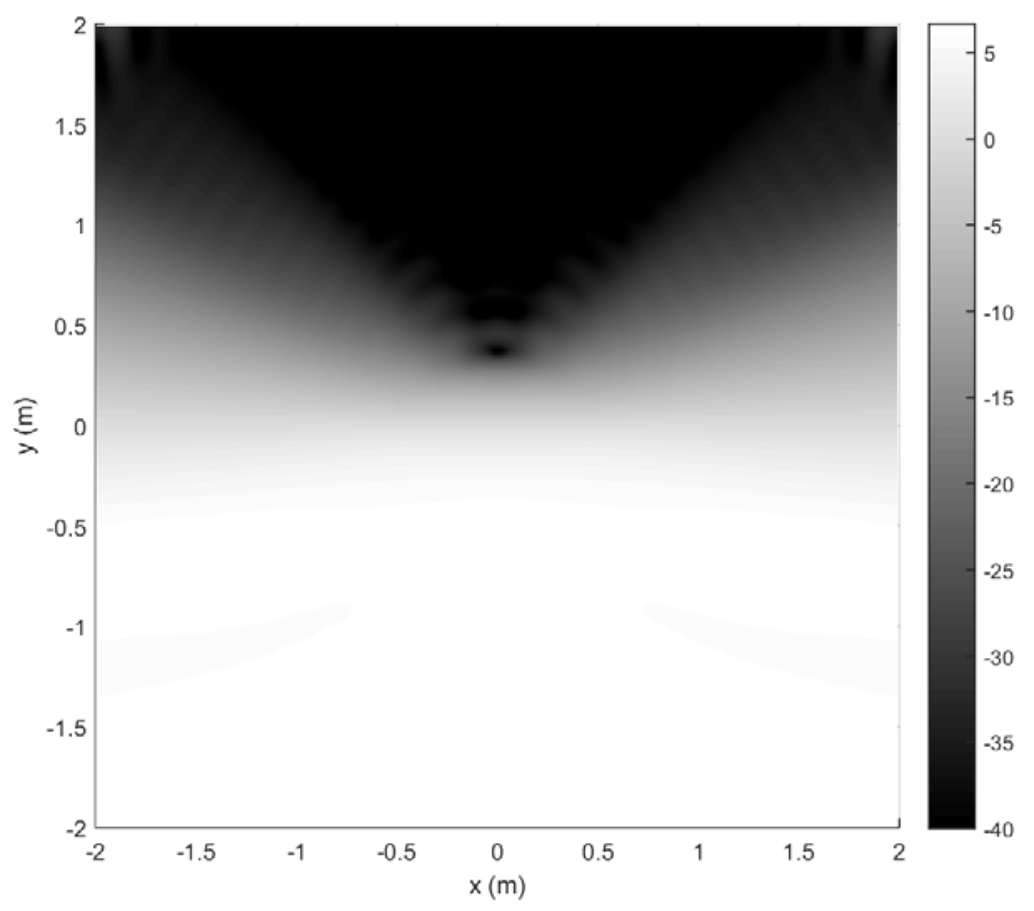

Figure 8: Half-space field in $\mathrm{dB}$ at $1 \mathrm{kHz}, \mathrm{N}_{\mathrm{T}}=15$ and offset 0 


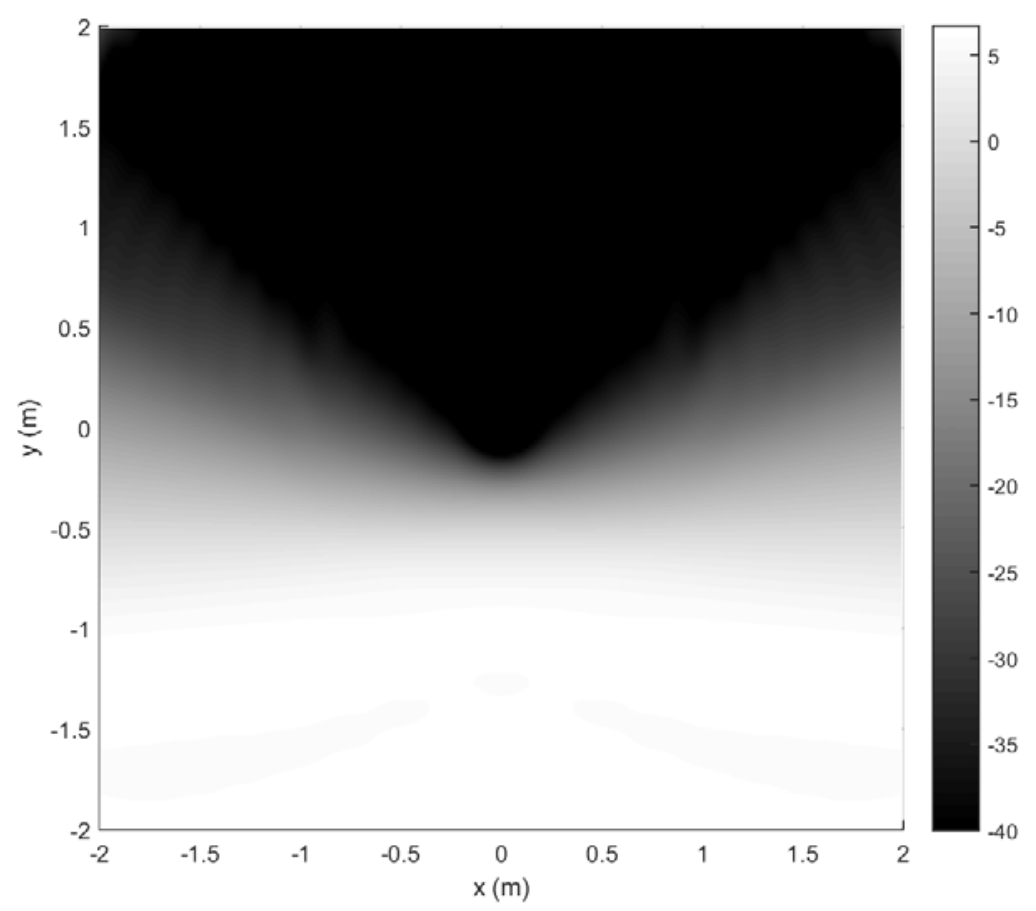

Figure 9: Half-space field in $\mathrm{dB}$ at $1 \mathrm{kHz}, \mathrm{N}_{\mathrm{T}}=15$ and offset 10

\section{Generation of 3D half-space sound fields at a single frequency}

It will now be briefly shown that a similar approach may be taken to producing half space fields in the 3D case, where the two half spaces are defined in spherical coordinates $\mathbf{r}=(r, \theta, \phi)$ for $\phi \in[0, \pi]$ and for $\phi \in[\pi, 2 \pi]$.

The general solution to the Helmholtz equation may be written as the 3D Herglotz wave function

$$
p(r, \theta, \phi, k)=\frac{1}{4 \pi} \int_{0}^{\pi} \int_{0}^{2 \pi} B\left(\theta^{\prime}, \phi^{\prime}\right) e^{i \mathbf{k} \cdot \mathbf{r}} \sin \theta^{\prime} d \theta^{\prime} d \phi^{\prime},
$$

where $B\left(\theta^{\prime}, \phi^{\prime}\right)$ is the plane-wave angular spectrum at wavenumber $k$. This can be expanded as

$$
B\left(\theta^{\prime}, \phi^{\prime}\right)=\sum_{n=0}^{\infty} \sum_{m=-n}^{n} \beta_{n}^{m} Y_{n}^{m}\left(\theta^{\prime}, \phi^{\prime}\right),
$$

where $\beta_{n}^{m}$ are the plane-wave expansion coefficients and the spherical harmonics are defined as 15

$$
Y_{n}^{m}(\theta, \phi)=\sqrt{\frac{(2 n+1)}{4 \pi} \frac{(n-|m|) !}{(n+|m|) !}} P_{n}^{|m|}(\cos \theta) e^{i m \phi},
$$


where $P_{n}^{m}($.$) is the associated Legendre function { }^{16}$. Substituting this and the spherical expansion for the plane wave ${ }^{16}$ into (27) yields

$$
p(r, \theta, \phi, k)=\sum_{n=0}^{\infty} \sum_{m=-n}^{n} i^{n} \beta_{n}^{m} j_{n}(k r) Y_{n}^{m}(\theta, \phi) .
$$

For a finite value of $k r$, the expansion can be truncated to a maximum order $N=\lceil k r\rceil$ in a similar manner to the $2 \mathrm{D}$ case ${ }^{17}$.

The expansion in (30) can be rearranged as

$$
p(r, \theta, \phi, k)=\sum_{m=-\infty}^{\infty} \sum_{n=|m|}^{\infty} i^{n} B_{n}^{m}(k) j_{n}(k r) Y_{n}^{m}(\theta, \phi) .
$$

An analytic sound field in the azimuthal angle $\phi$ may now be formed in the same manner as for the 2D case. Using the weighting (5), the analytic field can be written

$$
\hat{p}(r, \theta, \phi, k)=\sum_{m=-\infty}^{\infty} \mu_{m} \sum_{n=|m|}^{\infty} i^{n} B_{n}^{m}(k) j_{n}(k r) Y_{n}^{m}(\theta, \phi) Y_{n}^{m}\left(\theta_{i}, \phi_{i}\right)^{*} .
$$

For a single plane wave with an angle of incidence - and for a point source with source angle - in the $(x, y)$ plane this produces a lower half-space field. It also produces half space fields for source angles out of the $(x, y)$ plane. However, as the source elevation angle tends to 0 or 180 degrees the half space field tends to increasingly intrude into the other half space and for $\theta_{i}=0$ or $\theta_{i}=180$ degrees the sound field has only vertical incidence and a half space field is no longer possible. This may be demonstrated for the general plane wave case, for which

$$
B_{n}^{m}(k)=Y_{n}^{m}\left(\theta_{i}, \phi_{i}\right)^{*},
$$

by writing (32) in the equivalent cylindrical form

$$
\hat{p}(R, \phi, z, k)=e^{i k z \cos \theta_{i}} \sum_{m=-\infty}^{\infty} \mu_{m} i^{m} J_{m}\left(k R \sin \theta_{i}\right) e^{i m\left(\phi-\phi_{i}\right)} .
$$

From (34) it can be seen that for $\theta_{i}=0$ or $\theta_{i}=\pi$ the argument of the Bessel function is zero, the expansion order reduces to 0 and the plane wave becomes $\exp (i k z)$ or $\exp (-i k z)$, which is no longer a half space field.

For the point source case the behaviour is similar to that of the plane wave. For example, the sound field produced in the $(x, y)$ plane at a frequency of $1 \mathrm{kHz}$ for a point source at a radius of $6 \mathrm{~m}$ and at source angles $\theta_{s}=90$ and $\phi_{s}=45$ degrees and 
using the smoothed step function (23) with $N_{T}=15$ is shown in Fig. 10. The sound field is a half space field similar to that produced for the 2D case, for example as in Fig. (6).

For source angles near $\theta_{i}=0$ or 180 degrees the half space is no longer produced. For example, the magnitude of the complex sound pressure at $1 \mathrm{kHz}$ as a function of $y$ is shown in Fig. 11 for a point source at $\phi_{s}=0$ degrees azimuth and at angles in elevation from $\theta_{s}=5$ to 90 degrees. As $\theta_{s}$ approaches 0 the transition through $y=0$ is slower and the lower halfspace field increasingly intrudes into the upper halfspace.

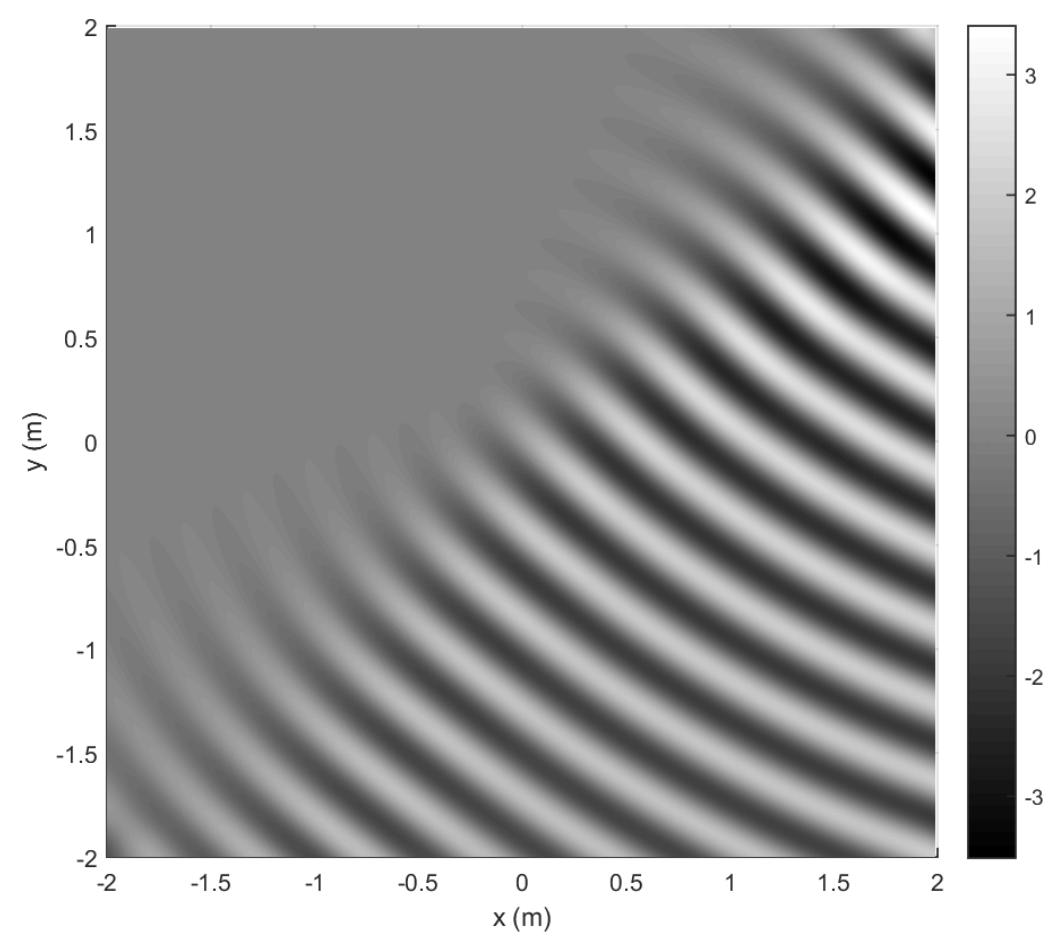

Figure 10: Half-space 3D field for $1 \mathrm{kHz}$ point source at $r_{\mathrm{s}}=6 \mathrm{~m}, \theta_{\mathrm{s}}=90$ and $\phi_{\mathrm{s}}=45$ degrees 


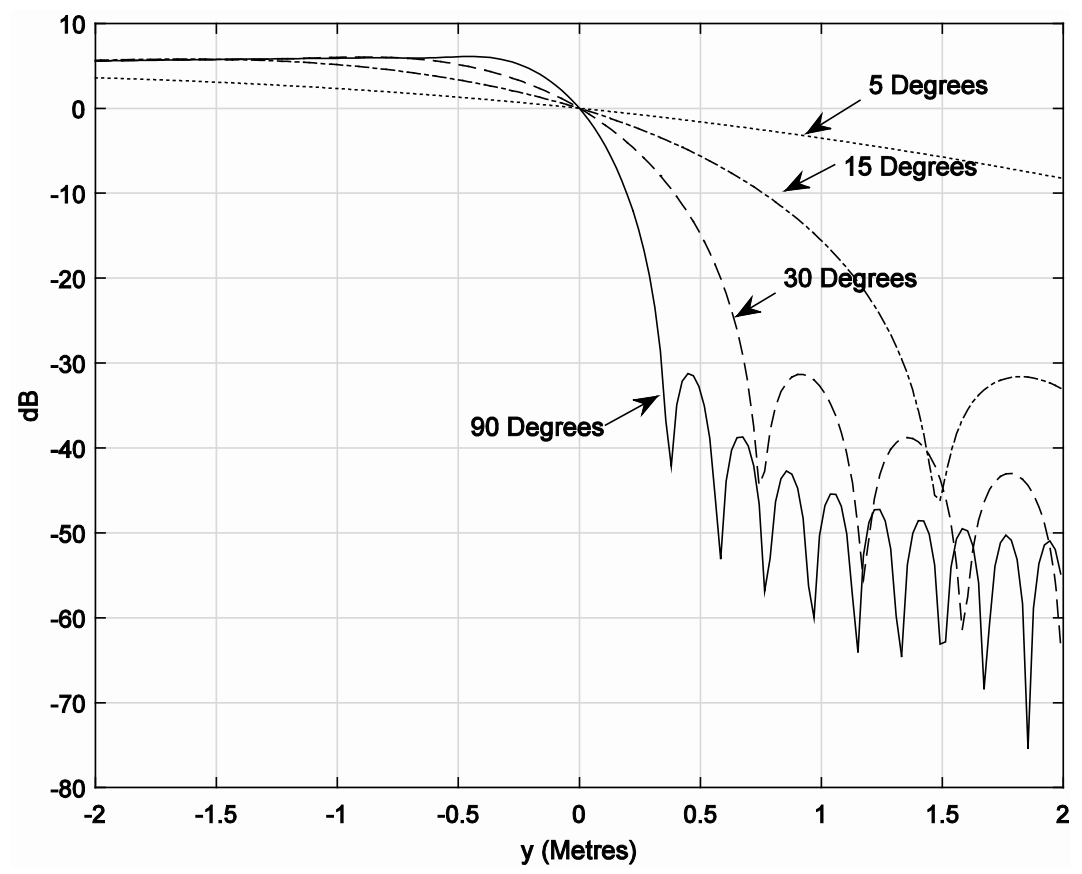

Figure 11: Half space sound pressure in $y$ for a $1 \mathrm{kHz}$ source at $\phi_{\mathrm{s}}=0$ degrees

\section{GENERATION OF WIDE BAND HALF-SPACE SOUND FIELDS}

The previous section outlined the generation of a half-space field at a single frequency. The extension of this approach to the wideband case is now considered. A simulation will be presented of the generation of a wide band pulse that traverses the upper or lower half-space within a circular loudspeaker array. For simplicity we consider only the 2D plane wave case.

Consider a circular array of 2D point sources at vector positions $\mathbf{r}_{l}$ with radius $\left\|\mathbf{r}_{l}\right\|=R_{L}$ and angles $\phi_{l}=2 \pi l / L, l \in[0, L-1]$. The spatial aliasing frequency of the array is 23

$$
f_{\text {alias }}=\frac{c(L-1)}{4 \pi R_{L}} .
$$

The array is able to reproduce with good accuracy a plane wave sound field up to $f_{\text {alias }}$ for all radii $R \leq R_{L}$.

Each point source produces a sound field inside the array of the form (24). The sum of these $L$ fields, weighted by amplitudes $w_{l}(k)$, are required to equal that of a general field with coefficients $\alpha_{m}{ }^{m}$, from Eq. (4), where $\alpha_{m}=1$ for a plane wave with angle of incidence equal to zero, weighted by $\mu_{m, N_{T}}$, from (23). The simple source solution for the weights is given by ${ }^{23}$ 


$$
w_{l}(k)=\frac{1}{L} \sum_{m=-M}^{M} \frac{\mu_{m, N_{T}} i^{m} e^{i m \phi_{l}}}{H_{m}\left(k R_{L}\right)} .
$$

The total field is then given by

$$
p(R, \phi)=\sum_{l=1}^{L} w_{l}(k) H_{0}\left(k\left|\mathbf{r}-\mathbf{r}_{l}\right|\right) .
$$

In the following example, a circular array of 351 sources at a radius of $2 \mathrm{~m}$ is used, producing a spatial aliasing frequency of $4.7 \mathrm{kHz}$. A sample rate of $f_{s}=8 \mathrm{kHz}$ is used and the signal length is $N=200$ samples, producing a time duration of $25 \mathrm{~ms}$. The weight solutions in Eq. (33) are generated for $N / 2=100$ equally-spaced positive frequencies $f_{q}=q f_{s} / N, q \in[1: N / 2]$ up to $4 \mathrm{kHz}$. The zero frequency weights, $q=0$, are set to zero. All computed loudspeaker weights $w_{l}(k)$ are multiplied by the linear phase term $\exp \left(-i \pi N f_{q} / f_{s}\right)$ to produce causal solutions with the main wave front arriving at the center of the array at time $12.5 \mathrm{~ms}$.

The complex sound field at each frequency, $p\left(R, \phi, k_{q}\right)$, is generated on a grid of 100 by 100 uniformly-spaced locations spanning an area of 4 by $4 \mathrm{~m}$.

In order to produce a similar half space transition width with frequency (see Fig. 3) a linear increase in transition width $N_{T}$ with frequency is used from a value $N_{T L}$ at low frequencies to $N_{T H}$ at high frequencies of the form

$$
N_{T}=N_{T L}+\frac{2 q}{N}\left(N_{T H}-N_{T L}\right), q \in[1, N / 2] .
$$

The simulations use $N_{T L}=5$ and $N_{T H}=35$.

To generate the physical sound field at a given time $t$, each spatial sound field $p\left(R, \phi, k_{q}\right)$ is multiplied by its corresponding temporal factor $\exp \left(i 2 \pi q f_{s} / N t\right)$ and summed to produce the complete field at time $t$. The real part of the total field is then plotted. For the array radius $R_{L}=2 \mathrm{~m}$ the propagation time of the pulse across the array is $\tau=2 R / c=11.8 \mathrm{~ms}$. The sound fields are produced for times $9.6 \mathrm{~ms}$, when the pulse is one quarter of the way across the array, at $12.5 \mathrm{~ms}$ when it is at the center, and at $15.4 \mathrm{~ms}$ at three-quarters of the distance across the array.

The sound fields at the three stated times are shown in Fig.s 12 to 14. Also shown are the lower half-space fields obtained using the complementary weights in Eq. (6). The pulses at $9.6 \mathrm{~ms}$ are reasonably well confined to their corresponding half-space, although there is a small residual curved wave front extending partially into the other 
half-space in each case. At $12.5 \mathrm{~ms}$ the pulses are well-confined to their half-space. At $15.4 \mathrm{~ms}$ a similar performance to that in Fig. 12 is seen.

(a)

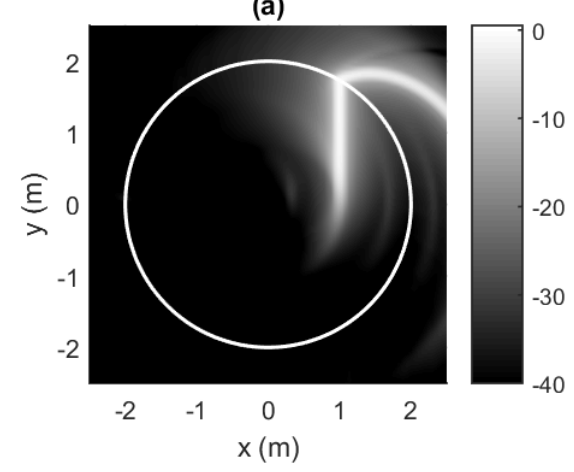

(b)

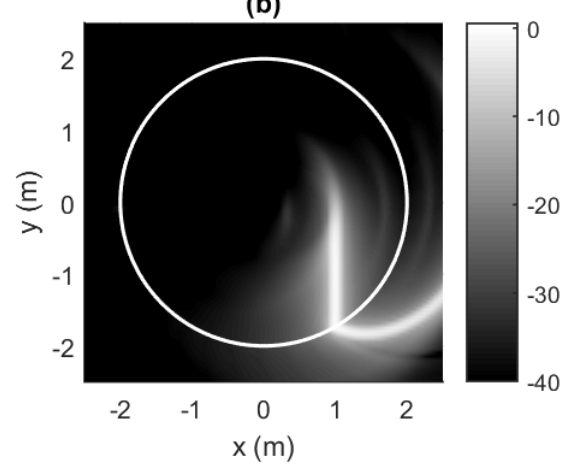

Figure 12: Sound pressure level of wideband pulse at time $9.6 \mathrm{~ms}$ for upper (a) and lower (b) half-space 
(a)

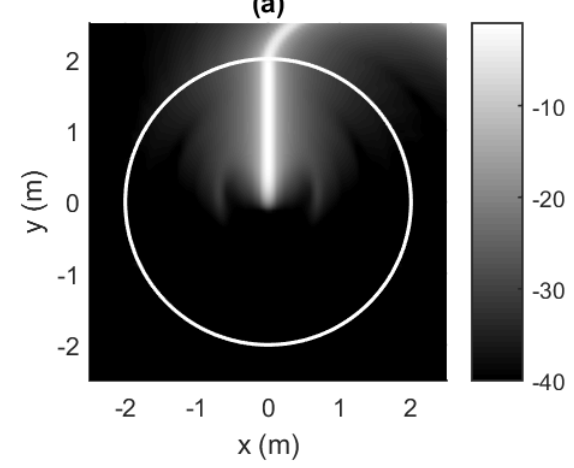

(b)

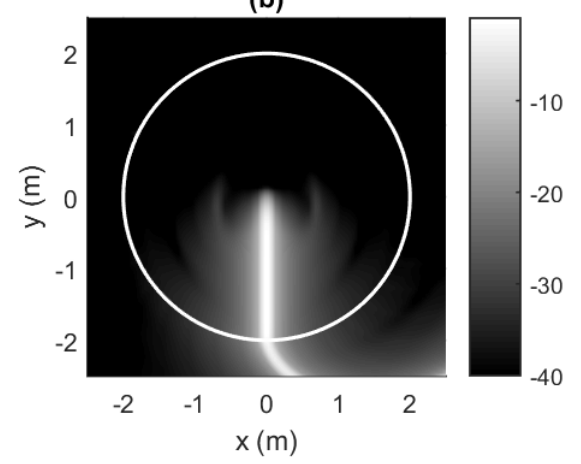

Figure 13: Sound pressure level of wideband pulse at time $12.5 \mathrm{~ms}$ for upper (a) and lower (b) half-space
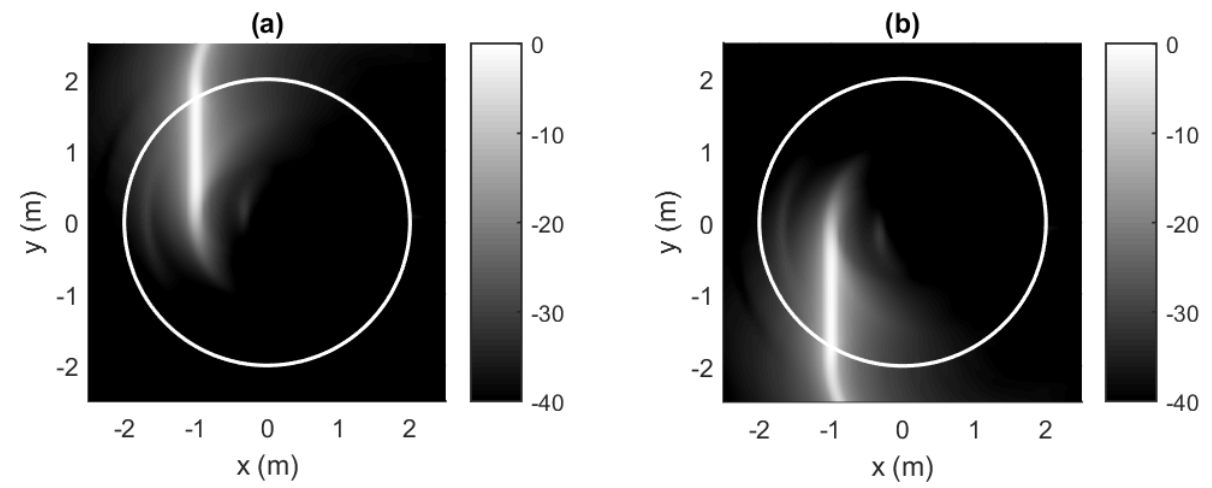

Figure 14: Sound pressure level of wideband pulse at time $15.4 \mathrm{~ms}$ for upper (a) and lower (b) half-space 
Finally, the two fields at time $12.5 \mathrm{~ms}$ are shown in Figure 15 for $L=81$ 2D point sources, which produces a spatial aliasing frequency of $1 \mathrm{kHz}$, one quarter of the Nyquist frequency. Compared to Figure 13, the sound field demonstrates pre- and post-oscillations in the reproduced pulse. However, the half-space field is maintained for radii less than $R_{M}=M / k_{\max }=0.54$ where $k_{\max }$ is the wavenumber at the Nyquist frequency $4 \mathrm{kHz}$.
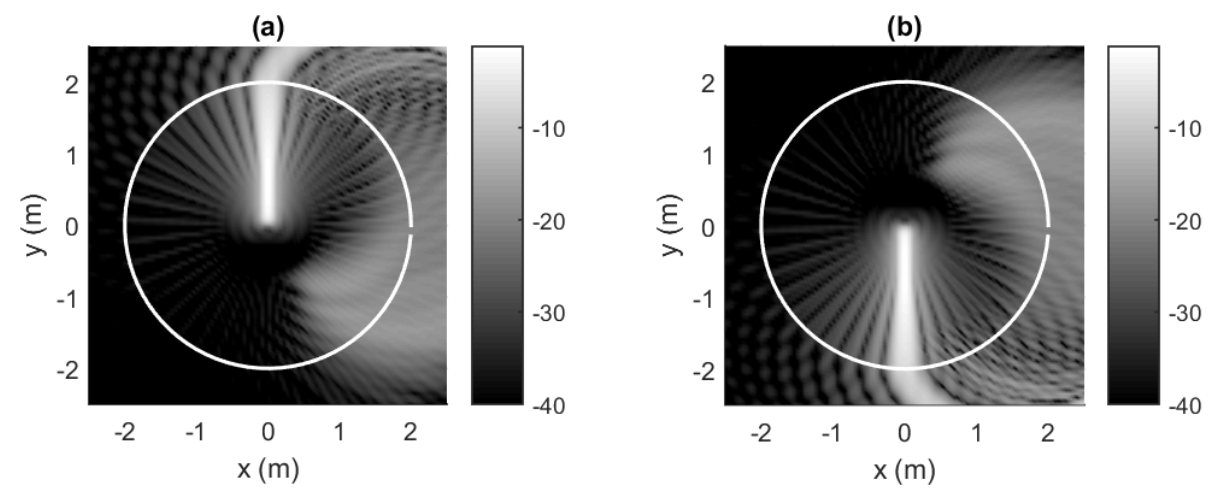

Figure 15: Sound pressure level of wideband pulse at time $12.5 \mathrm{~ms}$ for upper (a) and lower (b) half-space, using $\mathrm{L}=\mathbf{8 1} 2 \mathrm{D}$ sources and a corresponding spatial aliasing frequency of $1 \mathrm{kHz}$

\section{CONCLUSIONS}

This paper has shown that a sound field can be generated in a half-space without the need for a physical boundary, and reproduced using a circular array of loudspeakers. Half-space fields are produced by suppressing the positive or negative modes in the cylindrical or spherical harmonic sound field expansion. This operation is equivalent to adding to the sound field, in quadrature, a second field which is the Hilbert 
transform of the original sound field. The half space field may be equivalently termed an analytic sound field.

It has been shown that both plane wave and point source half-space fields can be produced. The use of a smooth transition between negative and positive mode weightings improves the accuracy of the half-space field and the extent of overlap between two half-space fields can be controlled by offsetting the weighting sequences. A simulation has been carried out to demonstrate the generation of wideband signals using a circular array of sources.

The advantage of the half-space approach is that two independent sound fields can be created over half the area within the array. The disadvantage is that the direction of sound propagation is limited to two directions parallel to the half-space boundary. However, in many applications for personal sound, a single direction of propagation is acceptable; for example when using line arrays. Furthermore it has been shown that it is possible to produce a field over less than 180 degrees in which a limited range of angles of arrival is produced allowing, for example, the reproduction of stereo sound over a confined region of the space.

Other techniques allow arbitrary directions of propagation, but when the direction of propagation in one zone is in-line with another zone, this produces interference. At low frequencies, it is possible to create a field that avoids intrusion into the other zone, but at high frequencies, the problem remains. Hence, the half space approach avoids interference between multiple zones by controlling the direction of propagation.

It has also been shown that a similar mode weighting approach applies for the 3D case, including the generation of half-space fields for out-of-plane sources, provided that the angle of arrival in elevation is not too close to 0 or 180 degrees (vertical incidence). However, since sound field expansions can be calculated under rotation of coordinates, it is likely that a half space field can be generated for any angle of arrival, although this has not been further investigated, or verified, here.

Finally, the simulations presented here have typically used a large number of sources and a sufficiently low sample rate to avoid spatial aliasing effects. An example of aliasing effects is given which shows that, within the reduced radius where reproduction is accurate, the half-space field is maintained. 


\section{ACKNOWLEDGEMENTS}

This work was supported in part by the Royal Academy of Engineering and by the Engineering and Physical Sciences Research Council.

\section{Appendix A}

The Fourier transform of the discrete signum function is

$$
S(\phi)=\sum_{m=-\infty}^{\infty} \operatorname{sgn}(m) e^{i m \phi}=\sum_{m=1}^{\infty} e^{i m \phi}-\sum_{m=1}^{\infty} e^{-i m \phi}
$$

This may be calculated by approximating the sum as

$$
\hat{S}(\phi)=\sum_{m=-\infty}^{\infty} e^{-\gamma|m|} \operatorname{sgn}(m) e^{i m \phi}=\sum_{m=1}^{\infty} e^{-[\gamma-i \phi] m}-\sum_{m=1}^{\infty} e^{-[\gamma+i \phi]}
$$

Taking out the first term of each summation and evaluating the geometric series

$$
\hat{S}(\phi)=\sum_{m=-\infty}^{\infty} e^{-\gamma|m|} \operatorname{sgn}(m)=e^{-\gamma}\left[\frac{e^{-[\gamma-i \phi]}}{1-e^{i \phi}}-\frac{e^{-[\gamma+i \phi]}}{1-e^{-i \phi}}\right]
$$

Letting $\gamma$ tend to zero yields

$$
S(\phi)=\frac{i \sin \phi}{1-\cos \phi}=\frac{i}{\tan (\phi / 2)}
$$

${ }^{1}$ W.F. Druyvesteyn and J. Garas, "Personal Sound," J. Audio Eng. Soc. 45, 685-701 (1997).

${ }^{2}$ T. Betlehem, W. Zhang, M.A. Poletti, and T.D. Abhayapala, "Personal sound zones," IEEE Signal Proc. Magazine 81-91 (2015).

${ }^{3}$ S. Baek, M.-S. Song, S.-P. Lee, and H.-G. Kang, "Speaker array system based on equalization method with a quiet zone," 127th AES Conv. (2009).

${ }^{4}$ M.A. Poletti, "An Investigation of 2D Multizone Surround Sound Systems," AES 125th Convention (2008).

${ }^{5}$ N. Radmanesh and I.S. Burnet, "Generation of isolated wideband sound fields using a combined two-stage lasso-LS algorithm," IEEE Trans. Audio, Speech Lang. Proc. 21, 378-387 (2013).

${ }^{6}$ Y.J. Wu and T.D. Abhayapala, "Spatial Multizone Soundfield Reproduction: Theory and Design," IEEE Trans. Audio, Speech Lang. Proc. 19, 1711-1720 (2011).

${ }^{7}$ J.-W. Choi and Y.-H. Kim, "Generation of an acoustically bright zone with an illuminated region using multiple sources," J. Acoust. Soc. Am. 111, 1695-1700 
(2002).

${ }^{8} \mathrm{~J} .-\mathrm{W}$. Choi and Y.-H. Kim, "Manipulation of sound intensity within a selected region using multiple sources," J. Acoust. Soc. Am. 116, 843-852 (2004).

${ }^{9}$ M. Shin, S.Q. Lee, F.M. Fazi, P.A. Nelson, D. Kim, S. Wang, K.H. Park, and J. Seo, "Maximization of acoustic energy difference between two spaces," J. Acoust. Soc. Am. 128, 121-131 (2010).

${ }^{10}$ J.-Y. Park, J.-H. Chang, and Y.-H. Kim, "Generation of independent bright zones for a two-channel private audio system," J. Audio Eng. Soc. 58, 382-393 (2010).

${ }^{11}$ P. Coleman, P. Jackson, M. Olik, and J.A. Pedersen, "Optimizing the planarity of sound zones," AES 52nd Intl. Conf. (2013).

${ }^{12}$ S.J. Elliott, J. Cheer, H. Murfet, and K.R. Holland, "Minimally radiating sources for personal audio," J. Acoust. Soc. Am. 128, 1721-1728 (2010).

${ }^{13}$ M. Jones and S.J. Elliott, "Personal audio with multiple dark zones," J. Acoust. Soc. Am. 124, 3497-3506 (2008).

${ }^{14}$ M.A. Poletti and F.M. Fazi, "An approach to generating two zones of silence with application to personal sound systems," J. Acoust. Soc. Am. 137, 598-605 (2015).

${ }^{15}$ D. Colton and R. Kress, Inverse Acoustic and Electromagnetic Scattering Theory (Springer, Berlin, 1998), p 13-37, p 54-63.

${ }^{16}$ E.G. Williams, Fourier Acoustics (Academic Press, San Diego, CA, 1999), p 115234.

${ }^{17}$ D.B. Ward and T.D. Abhayapala, "Reproduction of a plane-wave sound field using an array of loudspeakers," IEEE Trans. Speech and Audio Processing 9, 697 - 707 (2001).

${ }^{18} \mathrm{H}$. Boche and V. Pohl, "On the Calculation of the Hilbert Transform from Interpolated Data," IEEE Trans. Info. Theory 54, 2358-2366 (2008).

${ }^{19}$ L. Cohen, Time-Frequency Analysis, 1st ed. (Prentice-Hal, Inc., New Jersey, 1995), p 27-69.

${ }^{20}$ M. Abramowitz and I.A. Stegun, Handbook of Mathematical Functions with Formulas, Graphs and Mathematical Tables (National Bureau of Standards, 1972), p 361.

${ }^{21}$ A. Reilly, G. Frazer, and B. Boashash, "Analytic signal generation - tips and traps," IEEE Trans. Signal Proc. 42, 3241-3245 (1994).

${ }^{22}$ G.N. Watson, A Treatise on the Theory of Bessel Functions, 2nd ed. (Cambridge Univ. Press, New York, NY, 1995), p 358-372.

${ }^{23}$ M.A. Poletti and T.D. Abhayapala, "Interior and exterior sound field control using general two-dimensional first-order sources.," J. Acoust. Soc. Am. 129, 234-244 (2011). 


\section{Figure captions}

Figure 1: $\psi(R, \phi, k)$ for $k R=10$ and $k R=30$

Figure 2: Lower half-space field produced at $1 \mathrm{kHz}$ for a step function weighting

Figure 3: $\psi(R, \phi, k)$ for $k R=30, N_{T}=15$ transition width

Figure 4: Lower half-space field produced at $1 \mathrm{kHz}$ for $N_{T}=15$ transition width

Figure 5: Half-space field at $1 \mathrm{kHz}$ for three plane waves of equal amplitude with incidence angles $-30,0$ and 30 degrees, with $\mathrm{N}_{\mathrm{T}}=15$ and offset 10

Figure 6: Half-space field at $1 \mathrm{kHz}$ for a line source at $\left(R_{s}, \phi_{s}\right)=(4, \pi / 4), N_{T}=15$

Figure 7: Coefficients for a $1 \mathrm{kHz}$ half space field (a), Bessel coefficients (b) and the coefficients obtained by the convolution of the two for a translation of $-0.5 \mathrm{~m}$ in $\mathrm{y}$, (c)

Figure 8: Half-space field in $\mathrm{dB}$ at $1 \mathrm{kHz}, \mathrm{N}_{\mathrm{T}}=15$ and offset 0

Figure 9: Half-space field in $\mathrm{dB}$ at $1 \mathrm{kHz}, \mathrm{N}_{\mathrm{T}}=15$ and offset 10

Figure 10: Half-space 3D field for $1 \mathrm{kHz}$ point source at $\mathrm{r}_{\mathrm{s}}=6 \mathrm{~m}, \theta_{\mathrm{s}}=90$ and $\varphi_{\mathrm{s}}=45$ degrees

Figure 11: Half space sound pressure in $y$ for a $1 \mathrm{kHz}$ source at $\phi_{s}=0$ degrees

Figure 12: Sound pressure level of wideband pulse at time $9.6 \mathrm{~ms}$ for upper (a) and lower (b) half-space

Figure 13: Sound pressure level of wideband pulse at time $12.5 \mathrm{~ms}$ for upper (a) and lower (b) half-space

Figure 14: Sound pressure level of wideband pulse at time $15.4 \mathrm{~ms}$ for upper (a) and lower (b) half-space

Figure 15: Sound pressure level of wideband pulse at time $12.5 \mathrm{~ms}$ for upper (a) and lower (b) half-space, using $\mathrm{L}=81$ 2D sources and a corresponding spatial aliasing frequency of $1 \mathrm{kHz}$ 